\title{
ON THE MICROWAVE HOTSPOT PROBLEM
}

\author{
C. J. COLEMAN ${ }^{1,2}$
}

(Received 6 March 1990)

\begin{abstract}
When an object is heated by microwaves, isolated regions of excessive heating can often occur. The present paper investigates such hotspots by both perturbation and numerical means. For quite normal materials, it is shown that small temperature anomalies can grow to form hotspots. Furthermore, such effects do not need to be associated with thermal runaway.
\end{abstract}

\section{Introduction}

Microwave heating is by now a familiar technique in both industrial and domestic environments. Considering the large number of problems that it engenders, it is surprising that there has been little theoretical work on the thermal aspects (see [4] for a fairly comprehensive guide and [1, 2, 3 and 5] for more recent work). One of the more serious problems is the occurrence of hotspots. In this phenomenon, a localised temperature anomaly is magnified out of all proportion. A particularly dramatic example occurs during the microwave drying of bricks. In this case, a hotspot can cause the brick to explode [7]. The present paper explores the hotspot phenomenon, in order to provide a basis for possible remedies.

Microwave heating results from the dissipation of microwaves as they propagate through a body. For many materials, the rate of dissipation is temperature dependent, and this can dramatically affect the heating. As shown in Section 2 (and in reference [3]), several theoretical materials will show thermal runaway when subjected to uniform heating. Furthermore, as shown in Section 2, a small perturbation can explode, even when normalised by the

\footnotetext{
${ }^{1}$ Department of Mathematics, The University of Wollongong, Wollongong, NSW, 1500.

${ }^{2}$ Present address: Surveillance Research Laboratory, DSTO, PO Box 1650, Salisbury, S.A. 5108.

(C) Copyright Australian Mathematical Society 1991, Serial-fee code 0334-2700/91
} 
runaway background field. It should be noted, however, that realistic materials will only show the required absorption behaviour over a limited range of temperature. Consequently, the results must be treated with caution. Indeed, the simplest theories suggest that the absorption will moderate in a fashion that prevents runaway. Even so, it is found that a normalised perturbation can still reach extremely large values. This conclusion is supported by the numerical analysis of Section 4 in which the evolution of a localised temperature anomaly is simulated according to the full nonlinear theory.

\section{The uniform temperature field}

It is assumed that the dissipation of energy has negligible effect on the electromagnetic field and so the temperature distribution can be considered in isolation. For many materials, the absorption rate of energy is dependent on temperature [4] and so the thermal history will be described by an equation of the form

$$
\partial T / \partial t=\kappa \nabla^{2} T+f(T)
$$

where $\kappa$ is the thermal diffusivity and $f$ describes the energy absorption. For most microwave applications, the heating is so rapid that there is negligible heat loss through the surface of the body. Consequently, it is reasonable to assume zero heat flux at the boundaries. For a body with initially uniform temperature distribution, and uniform heating, this will result in a uniform distribution at later times with temperature evolving according to

$$
d T / d t=f(T) .
$$

Consider the exponential dissipation function $f=A \exp (a T)$ where $A$ and $a$ are positive. In this case, (2) will have the solution

$$
T(t)=-\ln (a C-a A t) / a
$$

where $C=\exp (-a T(0)) / a$.

It will be noted that (3) implies a temperature explosion at a finite time. Such a behaviour, however, is not a unique property of an exponential $f$. Indeed, it is a property of any $f(T)$ satisfying $f(T)>A T^{n}$ with $n>1$ and $A>0$. This can be seen from the inequality

$$
d T / d t>A T^{n}
$$

which can be integrated to yield

$$
\frac{1}{n-1}\left(T(0)^{1-n}-T^{1-n}\right)>A t \text {. }
$$


Rearranging (4),

$$
T(0)^{1-n}-A t(n-1)>T^{1-n}
$$

from which it is clear that the $T$ becomes infinite in a finite time.

The above solutions illustrate a runaway behaviour of the type that has been associated with hotspots [3]. It is unlikely, however, that the above forms of $f$ will be valid at large temperatures. Indeed, it is more likely that an Arrhenius type law $\left(f=f_{0} \exp (-E / R T)\right.$ with $f_{0}$ the limiting rate of dissipation) will be valid. Although the solution will no longer demonstrate a runaway behaviour, the next section shows that it can still exhibit hotspot type phenomena.

\section{Linear perturbations}

A possible explanation for the phenomenon of hotspots is the existence of thermal instabilities. Under such circumstances, a small local anomaly could blow up to form a hotspot. Consider the perturbation $T_{1}(\mathbf{r}, t)$ of a uniform temperature field $T_{0}(t)$. Temperature $T\left(=T_{0}+T_{1}\right)$ satisfies (1), from which

$$
\partial T_{1} / \partial t=\kappa \nabla^{2} T_{1}+T_{1} f^{\prime}\left(T_{0}\right)
$$

in the case of small perturbations. Consider a $T_{1}$ of the form

$$
T_{1}(\mathbf{r}, t)=\widehat{T}(\mathbf{r}) \stackrel{\circ}{T}(t) \text {. }
$$

From (5),

$$
\stackrel{\circ}{T}^{-1} \frac{\partial \stackrel{\circ}{T}}{\partial t}-f^{\prime}\left(T_{0}\right)=\kappa \nabla^{2} \widehat{T} / \widehat{T}=-\lambda
$$

where $\lambda$ is a constant. Obviously $\stackrel{\circ}{T}$ satisfies

$$
\stackrel{\circ}{T}^{-1} \frac{\partial \stackrel{\circ}{T}}{\partial t}-f^{\prime}\left(T_{0}\right)=-\lambda
$$

and $\widehat{T}$ satisfies

$$
\nabla^{2} \widehat{T}=-\lambda \widehat{T} / \kappa
$$

Furthermore, it should be noted that (6) will have the solution

$$
\stackrel{\circ}{T}=A f\left(T_{0}\right) \exp (-\lambda t) \text {. }
$$

The spatially uniform solution $T_{0}$ is considered to be unstable if $T_{1} / T_{0} \rightarrow$ $\infty$ in some temporal limit, this being the case if $\stackrel{\circ}{T} / T_{0} \rightarrow \infty$. From (7), however,

$$
\stackrel{\circ}{T} / T_{0} \propto\left(f\left(T_{0}\right) / T_{0}\right) e^{-\lambda t}
$$


and so, if the temperature blows up in a finite time, the material will be unstable if $f\left(T_{0}\right) / T_{0} \rightarrow \infty$ in that time. This is certainly the case if $f$ satisfies $f(T)>A T^{n} \quad(n>1$ and $A>0)$. Furthermore, this will also be the case for exponential $f$. Consequently, for a large class of materials, $T_{0}$ will be unstable in the linear sense. In such circumstances, small local anomalies in temperature will build up to form hotspots.

Although experimental studies [6] indicate that a great number of materials exhibit a temperature dependence for $f$ that could lead to the above instability, the available information is often limited in its temperature range and there is the likelihood that the dissipation will be moderated at elevated temperatures. Even so, it is possible for a large temperature differential to develop before the moderation comes into effect. To illustrate this, consider $\stackrel{\circ}{T} / T_{0}$ for the Arrhenius Law and a range of the parameter $\varepsilon=R T(0) / E$. Figure 1 shows some numerical results for $\varepsilon$ between 0.1 and $0.4(\lambda=0.2$ and $f_{0} / T(0)=1$ ), with (2) solved using a Runge-Kutta scheme. It will be noted that there is a peak in the temperature differential that is accentuated as $\varepsilon \rightarrow 0$.

As a particular example, consider a solid sphere (radius $a$ ) under the influence of the spherically symmetric perturbation $T_{1}=\widehat{T}(r) \stackrel{\circ}{T}(t) . \widehat{T}$ satisfies the differential equation

$$
\frac{d^{2} \hat{T}}{d r^{2}}+\frac{2}{r} \frac{d \hat{T}}{d r}+\frac{\lambda}{\kappa} \widehat{T}=0
$$

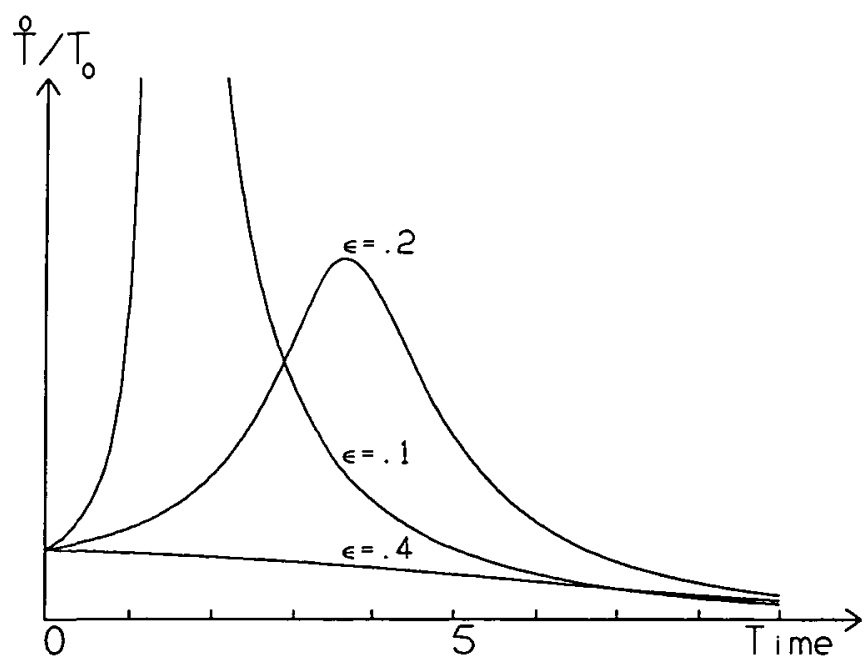

Figure 1. The normalised perturbation. 
which has bounded solutions of the form $\widehat{T}=\sin \left(\left(\frac{\lambda}{\kappa}\right)^{1 / 2} r\right) / r$. Parameter $\lambda$ satisfies

$$
a\left(\frac{\lambda}{\kappa}\right)^{\frac{1}{2}}-\tan \left(\left(\frac{\lambda}{\kappa}\right)^{\frac{1}{2}} a\right)=0
$$

in the case of an insulated boundary $(\partial \hat{T} / \partial r=0)$ at the surface $r=a$. Furthermore, the first four nontrivial solutions to (10) are given by $(\lambda / \kappa)^{\frac{1}{2}} a=$ $4.493,7.725,10.904$ and 14.066 . Since the higher-order modes will dampen out at a greater rate, it is obvious that any composite perturbation will tend to the lowest mode in the fullness of time. For present purposes, however, what happens in the short term is of greater interest. In particular, one must ask whether the normalised perturbation $\left(\stackrel{\circ}{T} / T_{0}\right)$ will peak. This occurs when $\partial\left(\stackrel{\circ}{T} / T_{0}\right) / \partial t=0$ which, together with (2) and (6), yields

$$
T_{0}\left(f^{\prime}\left(T_{0}\right)-\lambda\right)-f\left(T_{0}\right)=0 .
$$

For the Arrhenius law, this implies that $\bar{T}\left(=R T_{0} / E\right)$ satisfies

$$
\gamma-\exp \left(-\frac{1}{\bar{T}}\right)\left(\frac{1}{\bar{T}^{2}}-\frac{1}{\bar{T}}\right)=0 \text {. }
$$

where $\gamma=\lambda E / f_{0} R$. For $\gamma=0,0.1,0.2$, and 0.3 , (11) will have the solutions $0.1,0.7806,0.6154$ and 0.4341 . Above a value of about 0.305 for $\gamma$, the roots cease to exist. It is clear that $\gamma$ needs to be as large as possible for the peak to be eliminated, or at least moderated. Consideration of the spherical case suggests that this is equivalent to maximising $\kappa E /\left(L^{2} f_{0} R\right)$ where $L$ is a typical dimension of the heated body.

\section{Some numerical results}

Although the previous section suggests a possible mechanism for hotspot formation, the results should be treated with caution since they are based on a linear perturbation analysis. In order to provide additional evidence, the present section considers a numerical solution to the full problem. For a rectangular spacetime mesh with spatial intervals of length $h$ and time intervals of length $\tau$, an explicit finite difference discretisation of (1) is given by

$$
\begin{aligned}
& T_{i j k}^{l+1}=T_{i j k}^{l}+\alpha\left(T_{(i+1) j k}^{l}+T_{(i-1) j k}^{l}+T_{i(j+1) k}^{l}+T_{i(j-1) k}^{l}\right. \\
& \left.+T_{i j(k+1)}^{l}+T_{i j(k-1)}^{l}-6 T_{i j k}^{l}\right)+\tau f\left(T_{i j k}^{l}\right)
\end{aligned}
$$

where $T_{i j k}^{l}$ denotes the temperature at the space-time point $(i h, j h, k h, l \tau)$ 
and $\alpha=\kappa \tau / h^{2}$. Consider a cube with sides of length 2 and with centre located at the origin. Assume the sides to be insulated $(\partial T / \partial n=0)$ and that, at time $t=0$, there exists an isolated temperature anomaly of the form

$$
T(\mathbf{r})=(1+\delta \exp (-9 \mathbf{r} \cdot \mathbf{r}))
$$

Furthermore, assume $f$ to behave according to the Arrhenius law with $f(0)=0.1$. Figure 2 shows the behaviour of $\left(T-T_{0}\right) / T_{0}$ at the origin ( $T_{0}$ being the solution with $\delta=0$ ) for $\delta=0.1, \kappa=0.5$ and values of $\varepsilon$ between 0.1 and 0.4 . It will be noted that the behaviour is very similar to that predicted by the linear perturbation analysis. The development of the hotspot profile $(\varepsilon=.2)$ is shown in Figure 3. Here it will be noted that the hotspot phenomena is over before there is any significant dispersion of the initial perturbation profile. Similar conclusions arise when there are several hotspots, as illustrated in the profiles of Figure 4. This shows a situation with two additional hotspots on each coordinate axis, one on each side of the origin. For the above simulations, $\tau=0.002$ and $h=0.05$. The results, however, were validated over a range of these parameters.

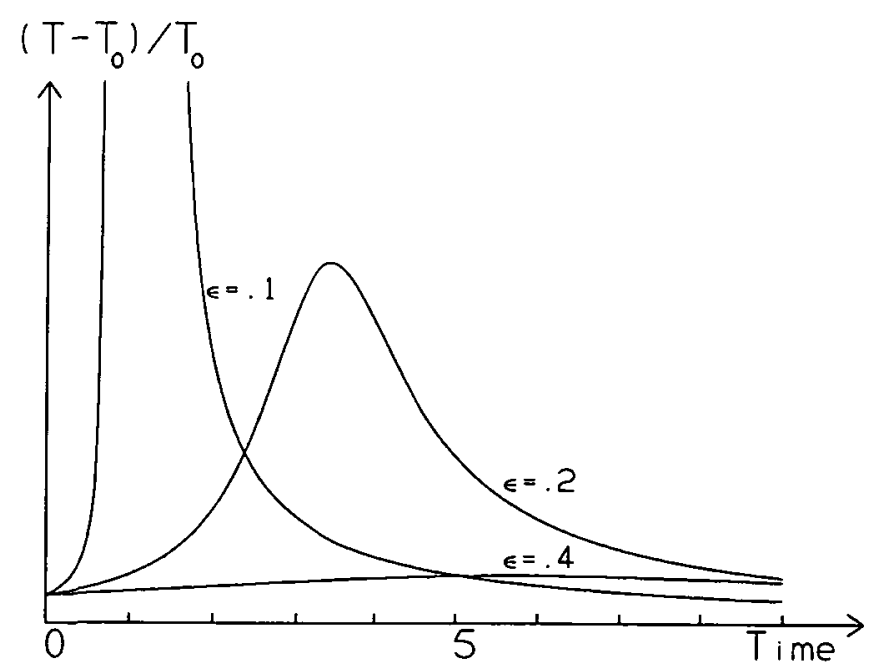

Figure 2. The normalised deviation from uniform heating. 


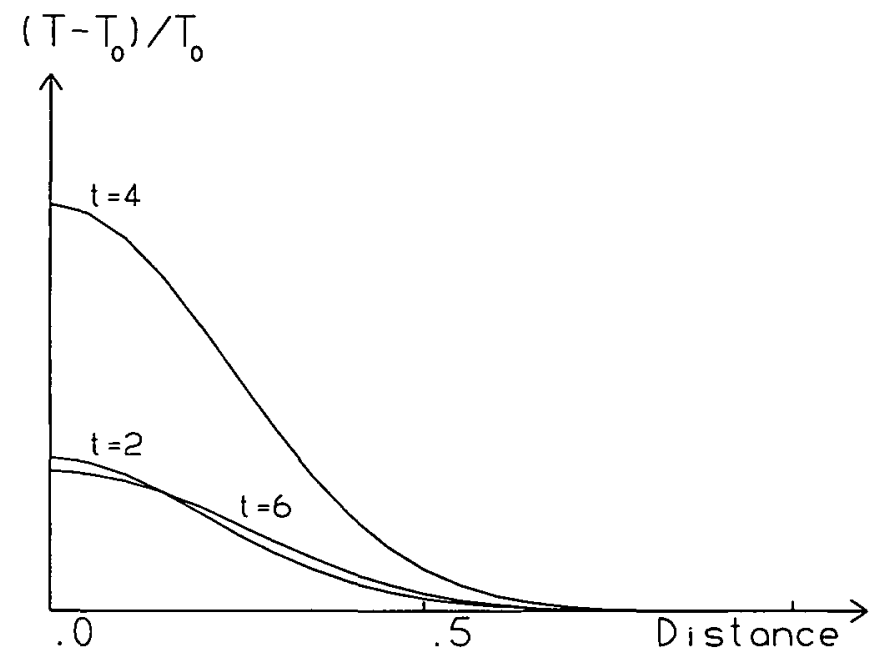

Figure 3. The hotspot profile.

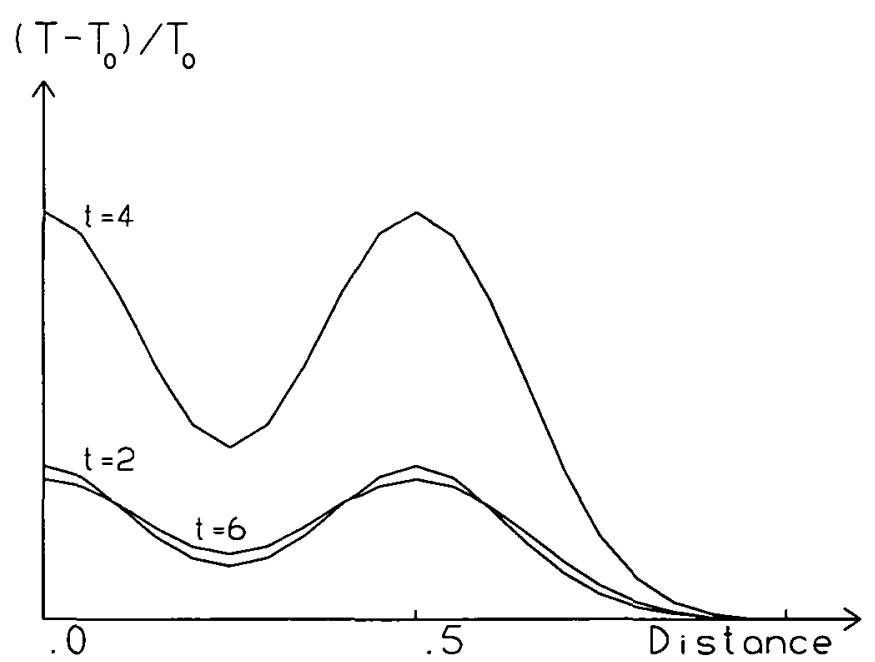

Figure 4. The multihotspot case.

\section{Conclusions}

It has been demonstrated that hotspot type phenomena can occur, even in materials that do not normally exhibit thermal runaway. Although such effects can be troublesome, there is now some evidence that they can also be used to advantage in the production of specialised metal alloys [7]. 
Consequently, it is important that further theoretical work be undertaken in order to aid the development of these processing techniques. In these circumstances, however, the dissipation is so strong that that interaction between the electromagnetic and thermal fields cannot be neglected. Consequently, future work will need to address this aspect.

\section{Acknowledgement}

The author would like to thank Dr. N. E. Smyth and Prof. J. M. Hill for helpful discussions.

\section{References}

[1] C. J. Coleman, "The microwave heating of frozen food substances", Applied Mathematical Modelling (1990).

[2] J. M. Hill, "Simple exact solutions applicable to microwave heating", ZAMP 40 (1989) 872-822.

[3] J. M. Hill and N. F. Smyth, "On the mathematical analysis of hotspots arising from microwave heating", Mathematical Engineering in Industry (1990).

[4] A. C. Metaxas and R. J. Meredith, "Industrial Microwave Heating", IEE Power Engineering Series, (Peregrinus, London, 1983).

[5] N. F. Smyth, "Microwave heating of bodies with temperature dependent properties", Wave Motion (1990).

[6] A. R. Von Hippel, “Dielectric Materials and Applications", (M.I.T. Press, Cambridge, Massachusetts, 1954).

[7] H. Worner, Private Communication. 